Check for updates

Cite this: Phys. Chem. Chem. Phys., 2020, 22, 129

Received 7th October 2019,

Accepted 1st November 2019

DOI: $10.1039 / c 9 c p 05473 a$

rsc.li/pccp

\section{About the origin of the large Stokes shift in aminoalkyl substituted heptamethine cyanine dyes $\dagger$}

\author{
Cristina Sissa, (D) *a Anna Painelli, (DD a Francesca Terenziani, (iD a Massimo Trotta (iD b \\ and Roberta Ragni iD *c
}

\begin{abstract}
Aminoalkyl-substituted heptamethine cyanine dyes are characterized by a large Stokes shift, an uncommon feature for cyanine molecules yet very promising for their application as fluorescent probes in bioimaging and as light harvesting antennas in biohybrid systems for solar energy conversion. The origin of this photophysical feature has not been adequately explored so far, and a combined experimental and theoretical work is herein provided to shed light on the role played by the central aminoalkyl substituent bound to the heptamethine cyanine backbone in defining the unusual properties of the dye. The spectra recorded in solvents of different polarities point to a marginal role of the medium in the definition of the Stokes shift, which conversely can be ascribed to the relaxation of the molecular geometry upon photoexcitation. This hypothesis is supported by an extensive theoretical investigation of the ground and excited states of the dye. TD-DFT results on the aminoalkyl-substituted dye and its unsubstituted precursor demonstrate a very similar cyanine-like structure for both molecules in the relaxed excited state. Conversely, in the ground state the amino substitution disrupts the conjugation in the polymethine chain, leading to a broken-symmetry, non-planar structure.
\end{abstract}

\section{Introduction}

Cyanines are a popular class of synthetic $\pi$-conjugated dyes characterized by a symmetric and charged structure with a polymethine chain bound to two peripheral electron donor (D) or acceptor (A) groups. The scientific interest towards cyanine dyes is mainly related to their spectral properties, such as high molar extinction coefficients and good fluorescence quantum yields, which make them excellent fluorescent probes for biological applications. ${ }^{1,2}$ The properties of cyanine dyes can be easily tuned by appropriately tailoring their chemical structure and functionalization by means of organic synthesis. The length of the polymethine chain and the nature of the peripheral groups can be modified to change the spectral position of absorption and emission bands. Furthermore, substituents can be designed to improve the solubility and/or provide chemical affinity to specific biotargets. ${ }^{3,4}$ Cyanines with short polymethine chains

\footnotetext{
${ }^{a}$ Dipartimento di Scienze Chimiche, della Vita e della Sostenibilità Ambientale, Università di Parma, Parco Area delle Scienze 17/A, 43124, Parma, Italy. E-mail: cristina.sissa@unipr.it

${ }^{b}$ CNR-IPCF, Institute for Physical and Chemical Processes, Bari unit, Via Orabona 4, 70125 Bari, Italy

"Dipartimento di Chimica, Università degli Studi di Bari "Aldo Moro",

I-, via Orabona 4, 70126 Bari, Italy. E-mail: roberta.ragni@uniba.it

$\dagger$ Electronic supplementary information (ESI) available. See DOI: 10.1039/c9cp05473a
}

typically show negligible solvatochromism, narrow absorption and emission bands with weak vibronic structures and very small Stokes shifts. ${ }^{5}$ On the opposite, cyanines with long polymethine chains are prone to symmetry breaking in the ground state, a phenomenon responsible for their unusual spectral properties: ${ }^{6-9}$ the position and the shape of absorption bands of long cyanines become strongly solvent-dependent, with broad and blue-shifted bands in polar solvents, while fluorescence bands maintain the weak solvatochromism and the vibronic structure typical of short cyanines. Essential-state models rationalize the spectral behaviour of both symmetry-preserving and symmetry-breaking cyanine dyes in a single theoretical framework. $8,10,11$

Heptamethine cyanines are promising for bioapplications; their spectra are in fact located in the near-infrared region, where the ability to penetrate biological tissues is the highest and the autofluorescence is marginal. Unfortunately, two main drawbacks hinder their use as fluorescent bioprobes: poor photostability and a very small Stokes shift (about $0.05 \mathrm{eV}$ ), the latter causing fluorescence self-absorption and quenching. Photostability is improved by introducing a chlorocyclohexenyl ring in the central position of the polymethine chain, ${ }^{12}$ and this also enhances the fluorescence quantum yield and provides a reactive site. This reactive site has been exploited to introduce a series of substituents on the central cyclohexenyl ring via nucleophilic substitution of the chlorine atom with amines, ${ }^{13}$ 
thiols, ${ }^{14-16}$ alcohols ${ }^{17,18}$ and phenyls. ${ }^{19,20}$ In particular, aminosubstituted heptamethine cyanine dyes obtained by substitution of the chlorine atom show a huge Stokes shift (larger than $0.3 \mathrm{eV}){ }^{13}$ maintaining or even improving the fluorescence properties typical of heptamethine dyes. These molecules have been exploited as fluorescent probes for bioimaging ${ }^{21,22}$ and in artificial light harvesting antennas for solar energy conversion. ${ }^{23-25}$

The mechanism at the basis of the spectroscopic effects observed upon substitution of the chlorine atom with an aminoalkyl group has not been fully addressed in the literature. Different research groups proposed an interplay between localized excitation and intermolecular charge transfer, but clear experimental evidence for this interplay is missing. ${ }^{13,23}$ The ion-pairing effect was also invoked for heptamethine cyanines showing different behaviours according to the counterion and/or the polarity of the solvent. ${ }^{19,26}$ The nitrogen group in the central core definitely affects the ground-state electronic configuration, favouring the presence of the charge on the central position. ${ }^{27,28}$ However, a thorough investigation of the first excited state and of its relaxation after vertical excitation is necessary to understand the origin of the large Stokes shift.

In this paper, a comparison between two cyanine dyes with different spectral properties is presented (Scheme 1): IR-780 is a commercially available dye bearing a chlorocyclohexenyl ring in the central position of the heptamethine cyanine structure, showing negligible ( $\approx 0.04 \mathrm{eV}$ ) Stokes shift; conversely HCy2, the amino-alkyl functionalized analogue obtained by nucleophilic substitution of the chlorine atom in IR-780 shows a large $(\approx 0.3 \mathrm{eV}$ ) Stokes shift. HCy2 has been selected and synthesized in the frame of studies on biohybrid systems for solar energy conversion based on the covalent bioconjugation of organic light harvesting antennas to photoenzymes. ${ }^{23-25}$ The specific octyl chain of HCy2 was chosen as a suitable spacer between the chromophore and the protein, in order to prevent the sterical hindrance of HCy2 on the photoenzyme surface, thus preserving the enzymatic photoactivity. ${ }^{29,30}$

A combined experimental and theoretical work sheds light on the origin of this large Stokes shift: the nitrogen atom linked to the cyclohexenyl ring strongly affects the conjugation of the polymethine chain, leading to a broken-symmetry ground state. On the opposite, the symmetry is partially restored in the first excited state (upon relaxation), leading to a large structural re-arrangement of the molecule that explains the observed large Stokes shift.

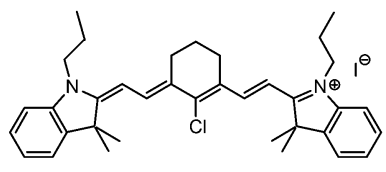

IR-780

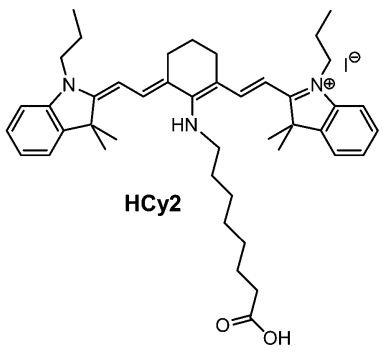

Scheme 1 Molecular structures of IR-780 and HCy2.

\section{Methods}

\section{Synthesis}

As reported in previous work, ${ }^{25}$ HCy2 was synthesized by reacting overnight IR-780 with 8-aminoottanoic acid in anhydrous $\mathrm{N}, \mathrm{N}$ dimethylformamide (DMF), under a nitrogen atmosphere at $80{ }^{\circ} \mathrm{C}$. DMF was then removed by dissolving the reaction mixture in dichloromethane and washing it with water. After drying the organic phase with anhydrous $\mathrm{Na}_{2} \mathrm{SO}_{4}$, dichloromethane was distilled at a reduced pressure and $\mathbf{H C y} 2$ was isolated as a blue solid in $48 \%$ yield by reverse phase C-18 column chromatography of the crude product using a mixture of water and acetonitrile $(6: 4 \mathrm{v} / \mathrm{v})$ as the mobile phase.

\section{Spectroscopic measurements}

Spectra grade or HPLC solvents were used to prepare solutions for absorption and emission measurements. Absorption spectra were recorded with a PerkinElmer Lambda650 spectrophotometer, while emission spectra were recorded with a FLS1000 Edinburgh Fluorometer equipped with a R5509-72 (Hamamatsu) NIR-PMT for detection in the near infrared region. Fluorescence spectra were recorded on dilute samples (with absorbance lower than 0.1). Fluorescence anisotropy spectra were recorded using a Horiba Jobin Ivon Fluoromax-3 instrument, equipped with polarizers. The sample was cooled using the Horiba FL-1013 Liquid Nitrogen Dewar assembly.

\section{Quantum chemical calculations}

All the reported quantum chemical calculations were performed using the Gaussian16 software. ${ }^{31}$ Ground-state geometry optimization was obtained at the DFT level of theory using $\omega \mathrm{B} 97 \mathrm{xD}$, B3LYP and M062X functionals and the 6-31g(d) basis set in toluene, DCM and DMSO, adopting the standard PCM model for solvation. Frequencies were computed after optimization using the same method. Vertical energies were calculated at the TD-DFT level of theory, adopting the same functionals and basis set. Solvation was accounted for in the framework of the corrected linear response theory. ${ }^{32,33}$ The relaxation of the first excited state towards its minimum energy was computed at the TD-DFT level, using the same functionals and basis set adopted for the ground state. Emission transition energies were calculated as vertical transitions from the relaxed vertical excited state (accounting for the corrected linear response theory for solvation).

\section{Results and discussion}

\section{Spectroscopic measurements}

The absorption and emission spectra recorded in solvents of different polarities (DCM and DMSO) are shown in Fig. 1 and the main data are summarized in Table 1. Absorption and fluorescence spectra of IR-780 are marginally affected by the solvent: the main absorption band is observed at 790 (796) $\mathrm{nm}$ in DCM (DMSO), and the Stokes shift is very small, with the emission maximum detected at 808 (816) $\mathrm{nm}$. Absorption and emission bands are narrow, with a weak vibronic shoulder in both solvents. The main absorption band of HCy2 at 665 (636) nm in 


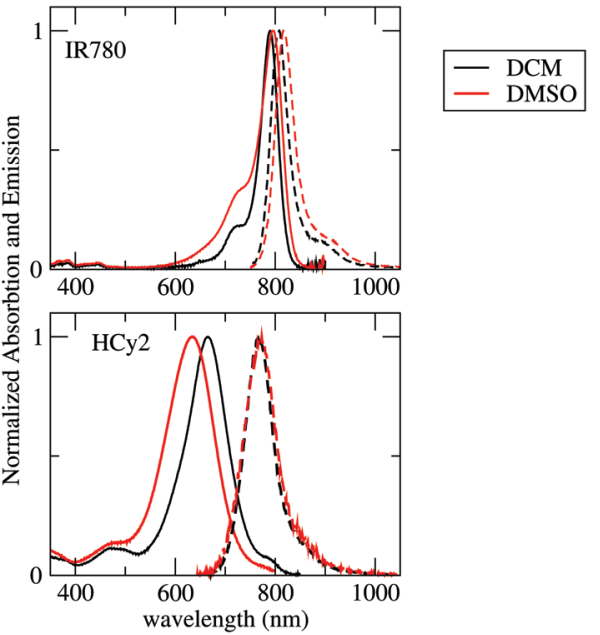

Fig. 1 Absorption (continuous lines) and emission (dashed lines) spectra of IR-780 and HCy2 in DCM (black lines) and DMSO (red lines).

Table 1 Experimental spectroscopic properties

\begin{tabular}{lllll}
\hline Molecule & Solvent & $\lambda_{\max }^{\mathrm{abs}}[\mathrm{nm}]$ & $\lambda_{\max }^{\mathrm{em}}[\mathrm{nm}]$ & Stokes shift $[\mathrm{eV}]$ \\
\hline IR-780 & DCM & 790 & 808 & 0.03 \\
& DMSO & 796 & 816 & 0.04 \\
HCy2 & DCM & 665 & 768 & 0.25 \\
& DMSO & 636 & 773 & 0.35
\end{tabular}

DCM (DMSO) is considerably blue-shifted with respect to that of IR-780, while the emission at $768(773) \mathrm{nm}$ is only slightly blue shifted with respect to that of IR-780. HCy2 shows a large Stokes shift, which increases with the solvent polarity from $0.25 \mathrm{eV}$ in DCM to $0.35 \mathrm{eV}$ in DMSO. HCy2 absorption bandshapes are broad, with an unresolved vibronic structure, while emission has a narrow bandshape, similar to that of IR-780. Neither IR-780 nor HCy2 are soluble in non-polar solvents.

Fluorescence anisotropy spectra provide details about the relative orientation of transition dipole moments of absorption and emission processes and therefore can be exploited to assess the possible contribution of different electronic excitations to the same absorption band, useful information when dealing with broad absorption spectra as for HCy2. The fluorescence excitation anisotropy spectrum recorded for HCy2 in glassy ethanol (EtOH) at $77 \mathrm{~K}$ is shown in Fig. 2. The fluorescence anisotropy signal is flat within the lowest excitation band, suggesting that a single electronic transition is responsible for the absorption band. The measured anisotropy is close to 0.3 , meaning that transition dipole moments of emission and absorption form an angle close to $24^{\circ}$. The Stokes shift measured at $77 \mathrm{~K}$ is somewhat reduced compared to that at room temperature (Fig. 2), but is still sizeable. This residual Stokes shift measured in the glassy solvent can be safely attributed to the relaxation of the molecular geometry after photoexcitation.

\section{Quantum chemical calculations}

DFT/TD-DFT calculations (see the Methods section for technical details) were performed to investigate the different spectroscopic

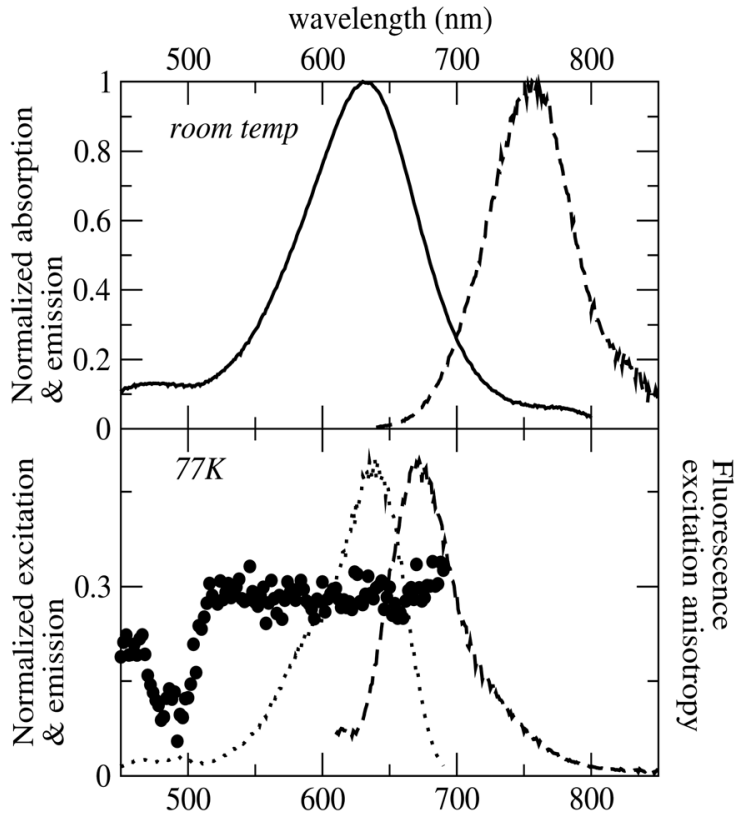

Fig. 2 Top panel: Absorption and emission of $\mathrm{HCy} 2$ at room temperature in $\mathrm{EtOH}$. Bottom panel: Excitation, emission and fluorescence excitation anisotropy spectra of $\mathrm{HCy} 2$ in $\mathrm{EtOH}$ at $77 \mathrm{~K}$.

behaviours of IR-780 and HCy2, and in particular to understand the origin of the large Stokes shift in HCy2. The ground state structure of both IR-780 and HCy2 was optimized in DCM and DMSO (adopting the PCM scheme at the DFT level of theory). Vertical excitations were calculated by TD-DFT adopting the corrected Linear Response theory for the solvent. ${ }^{32}$ Optimization of the first excited state was performed at the same level of theory. In this section, the results obtained with $\omega \mathrm{B} 97 \mathrm{xD}$ functional are discussed. Different functionals (B3LYP and M062X) were tested, providing qualitatively similar results (see the ESI $\dagger$ ), confirming the robustness of the approach. To speed up the calculation, without affecting the results, computations were performed on simplified molecular structures (Fig. 3), where the long alkyl chains are substituted by shorter groups. The carboxylic group in HCy2, (see Scheme 1) marginally affects its structural and optical properties, as discussed in the ESI. $\dagger$

The selected ground state properties of IR-780 and $\mathbf{H C y} \mathbf{2}$ are summarised in Table 2 (the dihedral angles $\delta_{1}$ and $\delta_{2}$ and the bond length alternations, $\mathrm{BLA}_{1}$ and $\mathrm{BLA}_{2}$ are defined in Fig. 3). The dihedral angles $\delta_{1}$ and $\delta_{2}$ provide information about the planarity of the molecule. The ground state of IR-780 has a planar structure, with $\delta_{1}$ and $\delta_{2}$ close to $180^{\circ}$. On the opposite, HCy2 is distinctly non-planar in the ground state, with both $\delta_{1}$ and $\delta_{2}$ appreciably different from $180^{\circ}$. Bond length alternations, BLA $_{1 / 2}$ are also calculated: a small BLA implies a good electronic delocalization along the chain (strong cyanine character), while a large BLA implies alternating single and double bonds. Moreover, dissimilar BLAs in the two molecular arms clearly point to symmetry breaking. In IR-780, $\mathrm{BLA}_{1}$ and $\mathrm{BLA}_{2}$ are both close to 0 , meaning that all bonds in the polymethine chain have a very similar character, intermediate between single and double bonds. $\mathrm{BLA}_{1}$ and $\mathrm{BLA}_{2}$ are larger in $\mathbf{H C y} \mathbf{2}$, with the bonds relevant 

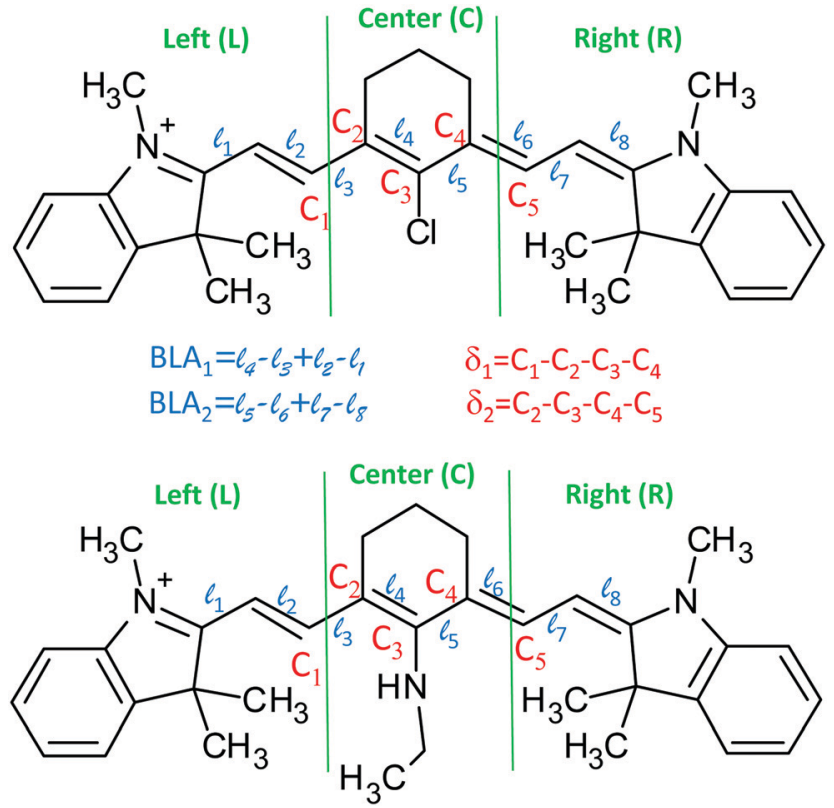

Fig. 3 Simplified molecular structures of IR-780 (top) and HCy2 (bottom) adopted for quantum chemical calculations. The dihedral angles, $\delta_{1}$ and $\delta_{2}$, and the bond length alternations, $B L A_{1}$ and $B L A_{2}$, are also defined. The green vertical lines mark the fragmentation of the molecules into a left and right arm and a central region.

Table 2 Selected ground state (GS) and the first excited state (1EX) properties of IR-780 and HCy2 in two different solvents

\begin{tabular}{llrlllll}
\hline Molecule & Solvent & $\delta_{1}\left({ }^{\circ}\right)$ & $\delta_{2}\left({ }^{\circ}\right)$ & $\mathrm{BLA}_{1}(\AA)$ & $\mathrm{BLA}_{2}(\AA)$ & $\ell_{4}(\AA)$ & $\ell_{5}(\AA)$ \\
\hline IR-780 (GS) & DCM & 174 & -174 & 0.015 & 0.015 & 1.408 & 1.408 \\
& DMSO & 174 & -174 & 0.015 & 0.015 & 1.407 & 1.407 \\
HCy2 (GS) & DCM & -162 & -154 & 0.110 & 0.086 & 1.444 & 1.434 \\
& DMSO & -162 & -154 & 0.113 & 0.089 & 1.445 & 1.436 \\
IR-780 (1EX) & DCM & 174 & -174 & 0.013 & 0.013 & 1.414 & 1.414 \\
& DMSO & 174 & -174 & 0.011 & 0.011 & 1.414 & 1.414 \\
HCy2 (1EX) & DCM & -173 & -170 & 0.025 & 0.024 & 1.420 & 1.422 \\
& DMSO & -174 & -171 & 0.023 & 0.022 & 1.419 & 1.422
\end{tabular}

to $\ell_{1}, \ell_{3}, \ell_{6}$ and $\ell_{8}$ having a predominant character of double bonds, and $\ell_{2}, \ell_{4}, \ell_{5}$ and $\ell_{7}$ having a predominant single-bond character. Furthermore, $\mathrm{BLA}_{1}$ differs from $\mathrm{BLA}_{2}$, pointing to a broken-symmetry ground state. The asymmetry is also confirmed by the length of the two central bonds of the polymethine chain $\left(\ell_{4}\right.$ and $\left.\ell_{5}\right)$ that in $\mathbf{H C y} 2$ are non-equivalent and significantly longer than in IR-780.

To discuss the charge distribution in the molecules, we have arbitrarily divided each molecule into three fragments (left, central and right), as defined in Fig. 3. The cumulative charges, calculated for the three fragments as the sum of Hirshfeld atomic charges, are given in Table 3. For IR-780, the charge is symmetrically distributed over the two molecular arms, and is mainly localized on the peripheral groups of the molecule. The structural asymmetry of $\mathbf{H C y} 2$ in the ground state shows up with a non-symmetric charge distribution in the two arms. Moreover, in HCy2 the charge residing in the central fragment is comparable to that in the peripheral subunits, in agreement with previous results by Pascal et al. ${ }^{27}$ The dyes are not soluble
Table 3 Ground-state and the first excited state (vertical and relaxed) charge distributions. The three subunits left, central and right are defined in (Fig. 3)

\begin{tabular}{lllll}
\hline Molecule & Solvent & Left & Central & Right \\
\hline IR-780 (GS) & DCM & 0.476 & 0.048 & 0.476 \\
HCy2 (GS) & DMSO & 0.464 & 0.072 & 0.464 \\
& DCM & 0.360 & 0.250 & 0.390 \\
IR-780 (1EX-Vertical) & DMSO & 0.322 & 0.322 & 0.356 \\
& DCM & 0.465 & 0.070 & 0.465 \\
HCy2 (1EX-Vertical) & DMSO & 0.439 & 0.122 & 0.439 \\
& DCM & 0.405 & 0.188 & 0.407 \\
IR-780 (1EX-Relaxed) & DMSO & 0.372 & 0.254 & 0.373 \\
& DCM & 0.464 & 0.072 & 0.464 \\
HCy2 (1EX-Relaxed) & DMSO & 0.441 & 0.118 & 0.441 \\
& DCM & 0.430 & 0.139 & 0.431 \\
& DMSO & 0.410 & 0.188 & 0.402 \\
\hline
\end{tabular}

in non-polar solvents, but calculations run in toluene (see the ESI $\dagger$ ) confirm that the solvent polarity marginally affects the ground state properties of either dye.

Table 4 summarizes the TD-DFT results for the lowest energy vertical excitation. The second electronic transition is calculated at much larger energies for both molecules, with an energy gap between the first and the second transition larger than $1.5 \mathrm{eV}$ for IR-780 and $0.9 \mathrm{eV}$ for HCy2. Therefore, the second electronic transition is irrelevant to the present discussion. The calculated transition energies are blue-shifted compared to the experiment, a well-known issue for cyanine dyes. ${ }^{34}$ The TD-DFT calculations in toluene (see the ESI $\dagger$ ) demonstrate negligible effects of solvent polarity on the absorption spectra of IR-780. The calculated absorption spectra in HCy2 slightly blue shift as the solvent polarity increases, in agreement with the experimental data. The lowest transition is well described as a HOMO-LUMO transition, and is delocalized over the whole molecule, as shown in Fig. 4. The HOMO and LUMO of IR-780 are perfectly symmetric, while the symmetry is lost in the orbitals of HCy2. The decrease of symmetry has important consequences in the energy positioning of the orbitals: the energy gap between the HOMO and LUMO is increased in HCy2, leading to a blue shift of the absorption band.

The geometry of the first excited state was optimized using the same level of theory adopted for the ground state and accounting for the solvent in the corrected linear response theory of PCM. The solvent polarity barely affects the emission energy of either IR-780 or HCy2 (Table 4). The main qualitative difference between the two molecules concerns the Stokes shift, which, in excellent agreement with the experiment, is predicted to be very small for IR-780 (less than $0.1 \mathrm{eV}$ ) and becomes large (more than $0.3 \mathrm{eV}$ ) in HCy2.

We are in the position to clarify the origin of the large Stokes shift observed for HCy2. The role of solvent polarity is marginal, since comparable Stokes shifts are calculated in DCM and DMSO (see Table 4) as well as in toluene (see the ESI†). The origin of Stokes shift is therefore safely ascribed to the geometry relaxation in the excited state.

Tables 2 and 3 summarize the computational results about the geometry and charge distribution in the vertical and relaxed excited states of the two molecules. Important structural 
Table 4 Calculated spectroscopic properties

\begin{tabular}{|c|c|c|c|c|}
\hline Molecule & Solvent & Absorption $[\mathrm{nm} / \mathrm{eV}]$ & Emission $[\mathrm{nm} / \mathrm{eV}]$ & Stokes shift $[\mathrm{eV}]$ \\
\hline \multirow[t]{2}{*}{ IR-780 } & DCM & $556 / 2.23(\mathrm{H}-\mathrm{L}$ 0.69; H-1 $\rightarrow \mathrm{L}+1-0.13)$ & 574/2.16 (H-L 0.69; H-1 $\rightarrow$ L+1 0.13) & 0.07 \\
\hline & DMSO & $556 / 2.23(\mathrm{H}-\mathrm{L} \quad 0.69 ; \mathrm{H}-1 \rightarrow \mathrm{L}+10.13)$ & $577 / 2.15(\mathrm{H}-\mathrm{L} \quad 0.69 ; \mathrm{H}-1 \rightarrow \mathrm{L}+1-0.12)$ & 0.08 \\
\hline \multirow[t]{2}{*}{ НСу2 } & DCM & $482 / 2.57(\mathrm{H}-\mathrm{L} \quad 0.67 ; \mathrm{H}-1 \rightarrow \mathrm{L}+1-0.16)$ & $554 / 2.24(\mathrm{H}-\mathrm{L} \quad 0.69 ; \mathrm{H}-1 \rightarrow \mathrm{L}+10.13)$ & 0.33 \\
\hline & DMSO & $481 / 2.58(\mathrm{H}-\mathrm{L} \quad 0.67 ; \mathrm{H}-1 \rightarrow \mathrm{L}+1-0.16)$ & $556 / 2.23(\mathrm{H}-\mathrm{L} 0.69 ; \mathrm{H}-1 \rightarrow \mathrm{L}+10.13)$ & 0.35 \\
\hline
\end{tabular}

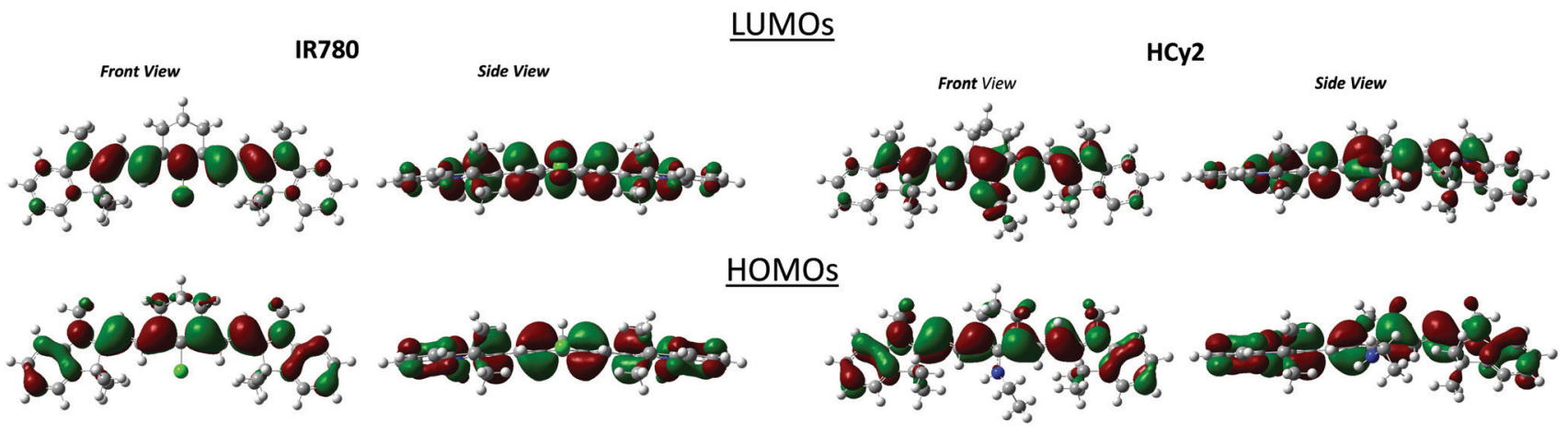

Fig. 4 The HOMO and LUMO of IR-780 (left) and HCy2 (right) in DMSO (equilibrium ground-state geometry).

changes upon excitation are observed for HCy2: (1) the dihedral angles $\delta_{1}$ and $\delta_{2}$ increase upon excitation and, becoming close to $180^{\circ}$, point to planarization of the molecule in the excited state; (2) both $\mathrm{BLA}_{1}$ and $\mathrm{BLA}_{2}$ significantly reduce and become comparable; and (3) the bond-lengths $\ell_{4}$ and $\ell_{5}$ also reduce and become comparable. Overall, the first excited state of $\mathbf{H C y} 2$ has a nearly planar and symmetric structure with an almost equalized bond-length, as a typical cyanine-like system. These structural changes in the excited state with respect to the ground state are responsible for the large observed Stokes shift in HCy2. On the opposite, no significant structural changes are observed in the first excited state of IR-780 with respect to the ground state, reflecting a small Stokes shift.

In HCy2, the charge distribution also undergoes significant changes in the excited state, as reported in Table 3. After excitation (the vertical excited state), the charge redistributes almost symmetrically over the two arms, and a significant charge depletion is observed in the central core of the molecule with respect to the ground state. The charge depletion in the central core of the molecule further increases after solvent relaxation, and in the relaxed excited state, charge distributions of $\mathbf{H C y} 2$ and IR-780 are very similar.

Quite interestingly, while important spectroscopic effects are observed upon substituting the central chlorine with aminogroups, the Stokes shift remains small in the case of substitution with thiols or alcohols. ${ }^{14,16-18}$ These experimental observations suggest that only the nitrogen atom has the ability to conjugate with the polymethine chain. From DFT calculations, in fact, the central $\mathrm{C}-\mathrm{N}$ bond shows an intermediate character between the single and double bond (the $\mathrm{C}-\mathrm{N}$ bond length in the ground state of the modified HCy2 is $\approx 1.35 \AA$ ). On the opposite, the DFT calculations on analogous structures with $-\mathrm{OCH}_{3}$ and $-\mathrm{SCH}_{3}$ substituents predict a predominant character of the single bond (see the ESI†).

\section{Conclusions}

The chemical structures of HCy2 and IR-780 heptamethine cyanine-dyes only differ in the nature of the unit bound to the central carbon atom of the polymethine chain: IR-780 bears a chlorine atom, whereas HCy2 an amino alkyl chain. This single substitution strongly affects the optical properties of the dyes, in particular their Stokes shift.

The absorption spectra of HCy2 are distinctly different from those of typical cyanine-dyes like IR-780. The absorption spectrum is strongly blue-shifted (more than $0.3 \mathrm{eV}$ ) and much broader compared to that of IR-780. On the opposite, the emission of HCy2 has a similar bandshape with respect to IR-780, and is only marginally blue-shifted (around $0.07 \mathrm{eV}$ ), resulting in a very large Stokes shift for HCy2. Long polymethine dyes are prone to symmetry breaking in the ground state: ${ }^{8}$ broken-symmetry cyanines show very broad absorption bands and a large Stokes shift, spectral features that are also recognized in HCy2. However, symmetry breaking in long polymethine dyes is typically induced by polar solvation, while absorption and fluorescence spectra of $\mathbf{H C y} 2$ are marginally affected by the solvent.

Quantum chemical calculations suggest that in the ground state of $\mathrm{HCy} 2$, the central C-N bond has an intermediate character between the single and double bonds. The presence of such a strong bond affects the geometry and conjugation of the polymethine chain: the ground state structure of $\mathbf{H C y} 2$ is non-planar and non-symmetric. In the ground state, the charge is preferentially localized in the central part of the molecule, ${ }^{27}$ and the charge distribution is not symmetric between the two molecular arms. Overall, looking at the ground state properties, HCy2 cannot be described as a cyanine-dye, but upon excitation, the molecule becomes a typical cyanine-dye: the relaxed excited state has an almost planar and symmetric structure, whose quasi-symmetric charge distribution resembles the charge distribution in IR-780. 
The origin of the Stokes shift of amino-substituted polymethine dyes is ascribed to the important structural changes occurring after photoexcitation. Symmetry breaking plays a key role in the observation of the large Stokes shift in symmetric structures: as experimentally observed and rationalized in terms of essential-state models, ${ }^{8,11}$ large Stokes shifts (strongly dependent on solvent polarity) are observed when symmetry breaking occurs in the first excited state or in the ground state. ${ }^{8,10}$ Here a different type of ground-state symmetry breaking is observed that originates from a structural distortion due to the strong conjugation of the amino group with the polymethine chain: this structural distortion does not depend on solvent polarity, and is almost lost in the first excited state, giving broad and blue-shifted absorption spectra, and cyanine-like narrow emission spectra.

\section{Conflicts of interest}

There are no conflicts to declare.

\section{Acknowledgements}

This work was supported by CINECA through the project IscrC_CANTA and from the HPC (High Performance Computing) facility of the University of Parma. C. S., A. P. and F. T. benefited from the equipment and framework of the COMP-HUB Initiative, funded by the 'Departments of Excellence' program of the Italian Ministry for Education, University and Research (MIUR, 20182022). C. S. thanks the University of Parma for financial support (Project Cybamat, FIL 2016 "Quota incentivante”). This project has been partly funded by the European Union Horizon 2020 research and innovation programme under grant agreement No. 812872 (TADFlife) and the European Union Horizon 2020 FET-Open project 800926-HyPhOE (Hybrid Electronics based on Photosynthetic Organisms).

\section{Notes and references}

1 A. Mishra, R. K. Behera, P. K. Behera, B. K. Mishra and G. B. Behera, Cyanines during the 1990s: a review, Chem. Rev., 2000, 100, 1973-2011.

2 J. L. Bricks, A. D. Kachkovskii, Y. L. Slominskii, A. O. Gerasov and S. V. Popov, Molecular design of near infrared polymethine dyes: A review, Dyes Pigm., 2015, 121, 238-255.

3 H. A. Shindy, Fundamentals in the chemistry of cyanine dyes: A review, Dyes Pigm., 2017, 145, 505-513.

4 M. Hervé, R. Brédy, G. Karras, B. Concina, J. Brown, A. R. Allouche, F. Lépine and I. Compagnon, On-the-Fly Femtosecond Action Spectroscopy of Charged Cyanine Dyes: Electronic Structure versus Geometry, J. Phys. Chem. Lett., 2019, 10, 2300-2305.

5 L. D. Patsenker, A. L. Tatarets and E. A. Terpetschnig, Long-Wavelength Probes and Labels Based on Cyanines and Squaraines, in Advanced Fluorescence Reporters in Chemistry and Biology I: Fundamentals and Molecular Design, ed. A. P. Demchenko, Springer Ser Fluoresc, 2010, vol. 8, pp. 65-104, DOI: 10.1007/978-3-642-04702-2_3.

6 L. M. Tolbert and X. Zhao, Beyond the cyanine limit: Peierls distortion and symmetry collapse in a polymethine dye, J. Am. Chem. Soc., 1997, 119, 3253-3258.

7 R. S. Lepkowicz, O. V. Przhonska, J. M. Hales, J. Fu, D. J. Hagan, E. W. Van Stryland, M. V. Bondar, Y. L. Slominsky and A. D. Kachkovski, Nature of the electronic transitions in thiacarbocyanines with a long polymethine chain, Chem. Phys., 2004, 305, 259-270.

8 F. Terenziani, O. V. Przhonska, S. Webster, L. A. Padilha, Y. L. Slominsky, I. G. Davydenko, A. O. Gerasov, Y. P. Kovtun, M. P. Shandura, A. D. Kachkovski, D. J. Hagan, E. W. Van Stryland and A. Painelli, Essential-state model for polymethine dyes: Symmetry breaking and optical spectra, J. Phys. Chem. Lett., 2010, 1, 1800-1804.

9 R. L. Gieseking, M. K. Ravva, V. Coropceanu and J. L. Brédas, Benchmarking Density Functional Theory Approaches for the Description of Symmetry Breaking in Long Polymethine Dyes, J. Phys. Chem. C, 2016, 120, 9975-9984.

10 F. Terenziani, A. Painelli, C. Katan, M. Charlot and M. BlanchardDesce, Charge instability in quadrupolar chromophores: Symmetry breaking and solvatochromism, J. Am. Chem. Soc., 2006, 128, 15742-15755.

11 C. Sissa, P. M. Jahani, Z. G. Soos and A. Painelli, Essential state model for two-photon absorption spectra of polymethine dyes, ChemPhysChem, 2012, 13, 2795-2800.

12 L. Strekowski, M. Lipowska and G. Patonay, Substitution Reactions of a Nucleofugal Group in Heptamethine Cyanine Dyes. Synthesis of an Isothiocyanato Derivative for Labeling of Proteins with a Near-Infrared Chromophore, J. Org. Chem., 1992, 57, 4578-4580.

13 X. Peng, F. Song, E. Lu, Y. Wang, W. Zhou, J. Fan and Y. Gao, Heptamethine cyanine dyes with a large Stokes shift and strong fluorescence: A paradigm for excited-state intramolecular charge transfer, J. Am. Chem. Soc., 2005, 127, 4170-4171.

14 S. A. Hilderbrand, K. A. Kelly, R. Weissleder and C. H. Tung, Monofunctional near-infrared fluorochromes for imaging applications, Bioconjugate Chem., 2005, 16, 1275-1281.

15 M. Helle, E. Rampazzo, M. Monchanin, F. Marchal, F. Guillemin, S. Bonacchi, F. Salis, L. Prodi and L. Bezdetnaya, Surface chemistry architecture of silica nanoparticles determine the efficiency of in vivo fluorescence lymph node mapping, ACS Nano, 2013, 7, 8645-8657.

16 F. Song, X. Peng, E. Lu, R. Zhang, X. Chen and B. Song, Syntheses, spectral properties and photostabilities of novel water-soluble near-infrared cyanine dyes, J. Photochem. Photobiol., A, 2004, 168, 53-57.

17 H. S. Choi, K. Nasr, S. Alyabyev, D. Feith, J. H. Lee, S. H. Kim, Y. Ashitate, H. Hyun, G. Patonay, L. Strekowski, M. Henary and J. V. Frangioni, Synthesis and in vivo fate of zwitterionic near-infrared fluorophores, Angew. Chem., Int. Ed., 2011, 50, 6258-6263.

18 Z. Zhang and S. Achilefu, Synthesis and evaluation of polyhydroxylated near-infrared carbocyanine molecular probes, Org. Lett., 2004, 6, 2067-2070. 
19 S. Mukhopadhyay, C. Risko, S. R. Marder and J. L. Brédas, Polymethine dyes for all-optical switching applications: A quantum-chemical characterization of counter-ion and aggregation effects on the third-order nonlinear optical response, Chem. Sci., 2012, 3, 3103-3112.

20 S. Zhang, P. Shao and M. Bai, In vivo type 2 cannabinoid receptor-targeted tumor optical imaging using a near infrared fluorescent probe, Bioconjugate Chem., 2013, 24, 1907-1916.

21 T. C. Wang, F. Cochet, F. A. Facchini, L. Zaffaroni, C. Serba, S. Pascal, C. Andraud, A. Sala, F. Di Lorenzo, O. Maury, T. Huser and F. Peri, Synthesis of the New Cyanine-Labeled Bacterial Lipooligosaccharides for Intracellular Imaging and in Vitro Microscopy Studies, Bioconjugate Chem., 2019, 30, 1649-1657.

22 Z. Shen, B. Prasai, Y. Nakamura, H. Kobayashi, M. S. Jackson and R. L. McCarley, A Near-Infrared, Wavelength-Shiftable, Turn-on Fluorescent Probe for the Detection and Imaging of Cancer Tumor Cells, ACS Chem. Biol., 2017, 12, 1121-1132.

23 S. la Gatta, F. Milano, G. M. Farinola, A. Agostiano, M. Di Donato, A. Lapini, P. Foggi, M. Trotta and R. Ragni, A highly efficient heptamethine cyanine antenna for photosynthetic Reaction Center: From chemical design to ultrafast energy transfer investigation of the hybrid system, Biochim. Biophys. Acta, Bioenerg., 2019, 1860, 350-359.

24 R. Ragni, G. Leone, G. Rizzo, S. la Gatta, F. Milano, M. Trotta and G. M. Farinola, Synthesis of two cyanine dyes as potential artificial antennas for the bacterial photosynthetic Reaction Center, MRS Adv., 2019, 4, 1293-1298.

25 R. Ragni, G. Leone, S. la Gatta, G. Rizzo, M. Lo Presti, V. De Leo, F. Milano, M. Trotta and G. M. Farinola, A heptamethine cyanine dye suitable as antenna in biohybrids based on bacterial photosynthetic reaction center, MRS Adv., 2019, 4, 1143-1148.

26 P. A. Bouit, C. Aronica, L. Toupet, B. Le Guennic, C. Andraud and O. Maury, Continuous symmetry breaking induced by ion pairing effect in heptamethine cyanine dyes: Beyond the cyanine limit, J. Am. Chem. Soc., 2010, 132, 4328-4335.

27 S. Pascal, A. Haefele, C. Monnereau, A. Charaf-Eddin, D. Jacquemin, B. Le Guennic, C. Andraud and O. Maury, Expanding the polymethine paradigm: Evidence for the contribution of a bis-dipolar electronic structure, J. Phys. Chem. A, 2014, 118, 4038-4047.

28 B. Le Guennic and D. Jacquemin, Taking Up the Cyanine Challenge with Quantum Tools, Acc. Chem. Res., 2015, 48, 530-537.
29 F. Milano, R. R. Tangorra, O. Hassan Omar, R. Ragni, A. Operamolla, A. Agostiano, G. M. Farinola and M. Trotta, Enhancing the light harvesting capability of a photosynthetic reaction center by a tailored molecular fluorophore, Angew. Chem., Int. Ed., 2012, 51, 11019-11023.

30 O. Hassan Omar, S. La Gatta, R. R. Tangorra, F. Milano, R. Ragni, A. Operamolla, R. Argazzi, C. Chiorboli, A. Agostiano, M. Trotta and G. M. Farinola, Synthetic Antenna Functioning As Light Harvester in the Whole Visible Region for Enhanced Hybrid Photosynthetic Reaction Centers, Bioconjugate Chem., 2016, 27, 1614-1623.

31 M. J. Frisch, G. W. Trucks, H. B. Schlegel, G. E. Scuseria, M. A. Robb, J. R. Cheeseman, G. Scalmani, V. Barone, G. A. Petersson, H. Nakatsuji, X. Li, M. Caricato, A. V. Marenich, J. Bloino, B. G. Janesko, R. Gomperts, B. Mennucci, H. P. Hratchian, J. V. Ortiz, A. F. Izmaylov, J. L. Sonnenberg, D. Williams-Young, F. Ding, F. Lipparini, F. Egidi, J. Goings, B. Peng, A. Petrone, T. Henderson, D. Ranasinghe, V. G. Zakrzewski, J. Gao, N. Rega, G. Zheng, W. Liang, M. Hada, M. Ehara, K. Toyota, R. Fukuda, J. Hasegawa, M. Ishida, T. Nakajima, Y. Honda, O. Kitao, H. Nakai, T. Vreven, K. Throssell, J. A. Montgomery, Jr., J. E. Peralta, F. Ogliaro, M. J. Bearpark, J. J. Heyd, E. N. Brothers, K. N. Kudin, V. N. Staroverov, T. A. Keith, R. Kobayashi, J. Normand, K. Raghavachari, A. P. Rendell, J. C. Burant, S. S. Iyengar, J. Tomasi, M. Cossi, J. M. Millam, M. Klene, C. Adamo, R. Cammi, J. W. Ochterski, R. L. Martin, K. Morokuma, O. Farkas, J. B. Foresman and D. J. Fox, Gaussian 16, Revision B.01, Gaussian, Inc., Wallingford CT, 2016.

32 M. Caricato, B. Mennucci, J. Tomasi, F. Ingrosso, R. Cammi, S. Corni and G. Scalmani, Formation and relaxation of excited states in solution: A new time dependent polarizable continuum model based on time dependent density functional theory, J. Chem. Phys., 2006, 124, 124520.

33 C. A. Guido, D. Jacquemin, C. Adamo and B. Mennucci, Electronic Excitations in Solution: The Interplay between State Specific Approaches and a Time-Dependent Density Functional Theory Description, J. Chem. Theory Comput., 2015, 11, 5782-5790.

34 D. Jacquemin, Y. Zhao, R. Valero, C. Adamo, I. Ciofini and D. G. Truhlar, Verdict: Time-dependent density functional theory 'not guilty' of large errors for cyanines, J. Chem. Theory Comput., 2012, 8, 1255-1259. 